Renata Szczepaniak (Hamburg)

\title{
Sprachwandel und sprachliche Unsicherheit \\ Der formale und funktionale Wandel des Genitivs seit dem Frühneuhochdeutschen
}

\begin{abstract}
Aus der Perspektive der Sprachbenutzerinnen ist der Genitiv vom Sprachverfall bedroht. Jedoch lässt sich in der Geschichte des Deutschen kein geradliniger Abbau nachweisen. Die kurze Genitivendung $-s$ (aus -es) setzte sich zwar schon im Frühneuhochdeutschen als die häufigere Variante durch, im weiteren Sprachwandel entwickelte sich dann aber eine komplex gesteuerte Variation beider Endungen. Mit dem Abbau des verbalen und attributiven Genitivs gehen zwar wichtige Funktionsbereiche verloren, doch zeichnet sich in der neuesten Sprachgeschichte ein unerwarteter Aufbau des Genitivs als Präpositionalkasus ab. In diesem Beitrag wird dafür plädiert, dass die formale und funktionale Entwicklung des Genitivs stark durch sprachliche Unsicherheit beeinflusst wurde und wird, die eine Reaktion auf bestehende Varianz darstellt. Es wird dafür argumentiert, dass die stilistische Aufwertung der langen Genitivform und des Genitivs gegenüber dem Dativ den Sprachwandel aufhält bzw. sogar in eine andere Richtung lenkt.
\end{abstract}

\section{Einleitung}

Der Genitiv, v.a. in seiner maskulinen und neutralen Singularform, gehört aus der Perspektive der Sprachbenutzer/innen zu den vom Sprachverfall bedrohten grammatischen Phänomenen. Tatsächlich lässt sich in der Geschichte des Deutschen ein formaler und funktionaler Wandel des Genitivs beobachten, jedoch kein geradliniger Abbau. Dies ist auf die Wechselwirkung zwischen Sprachwandel und sprachlicher Unsicherheit zurückzuführen. Der sich vollziehende Sprachwandel schafft auf der Systemebene Varianz, die auf der Sprachgebrauchsebene zu Unsicherheit führt. Diese wiederum kann den Sprachwandel blockieren oder sogar in eine andere Richtung lenken, wenn die (mehr oder weniger freien) Varianten in die Konstruktion von sozialen Kontrasten aufgenommen werden. In diesem konkreten Fall des formalen und funktionalen Wandels des Genitivs ist die bis heute bestehende Variation seit der Herausbildung der überregionalen Schriftsprache Gegenstand der Sprachreflexion und auch der polarisierenden und (explizit oder implizit) stigmatisierenden Sprachkritik. Es ist ein bewusstes und salientes Sprachwandelphänomen und zugleich ein „Vorzeigemodell“ für so genannten Sprachverfall. 
Dieser Artikel beschäftigt sich mit der Frage, inwieweit der unsichere Sprachbenutzer in den Sprach- und Normwandel eingreifen kann. Zum einen (Abschnitt 2) wird es um den formalen Normwandel von der langen zur kurzen Genitivendung gehen. Die formale Variation zwischen -es und $-s$ (z.B. des Fluges/des Flugs) resultiert aus der typologischen Tendenz des Deutschen zur Wortsprachlichkeit, die gerade im Frühneuhochdeutschen eine Phase der Intensivierung erfuhr. Im Zuge des typologischen Umbaus wurden Wortstrukturen phonologisch profiliert, indem die unbetonten Reduktionsvokale abgebaut und dadurch (passiv) die betonten Silben hervorgehoben wurden. Die Reduktion der Genitivform -es $>-s$ ist ein Teil dieser Entwicklung, die, stark zunächst vom prosodisch-phonologischen Kontext abhängig, schon im Mittelhochdeutschen begann. Doch fiel das verstärkte Aufkommen der kurzen Genitivendung in die frühneuhochdeutsche Phase, die für den Normwandel entscheidend war.

Auf der Systemebene war das Frühneuhochdeutsche die Phase des formalen Umbaus. Gleichzeitig war es die Zeit der Herausbildung der Standardsprache und damit die Zeit der Spracharbeit (siehe u.a. Hundt 2000). Auf die Existenz der funktional nicht unterscheidbaren Varianten -es und $-s$ reagierten die Grammatiker des 18. und 19. Jahrhunderts mit Polarisierung und Stigmatisierung der kurzen Genitivendung, die sich in gegenwärtigen Sprecherurteilen fortsetzen. Die mit dem Sprachwandel in Bewegung gesetzte systembezogene Instabilität erzeugt eine sprachliche Unsicherheit, die mit Hilfe soziolinguistischer Kriterien scheinbar stabilisier- bzw. stratifizierbar ist. Es steht zu vermuten, dass der formale Abbau der Genitivendung durch stilistische Kontrastierung, die in den Dienst der intendierten sozialen Verortung, eigentlich des sozialen Aufstiegs des Sprechers, gestellt wird, seit Jahrhunderten aufgehalten wird.

Zum anderen (Abschnitt 3) wird der funktionale Wandel betrachtet, der neben dem Abbau des verbalen und attributiven Genitivs einen Aufbau des präpositionalen Genitivs umfasst. Laienlinguistische Salienz erlangte der präpositionale Genitiv bereits im Frühneuhochdeutschen mit der Stigmatisierung der Dativrektion nach der Präposition wegen (siehe Davies/Langer 2006). Diese hallt in der populären Sprachkritik nach. Den Sprechern dient dieser Fall offensichtlich als (aus sprachhistorischer Perspektive inkorrektes) Muster zum Umgang mit schwankender Präpositionalrektion von sekundären Präpositionen, z.B. dank dem guten Wetter/dank des guten Wetters. Mit dem Prestigegenitiv können Fälle sprachlicher Unsicherheit gelöst werden. Als „Nebeneffekt" wird der Sprach- und Normwandel beeinflusst. 


\section{Von der langen zur kurzen Genitivendung}

Auf den ersten Blick ließen sich die kurze und die lange Genitivendung wie in des Fluges/des Flugs, des Korbes/des Korbs als Zweifelsfall bezeichnen. Als solcher gilt nach Klein (2003, S. 7) ,eine sprachliche Einheit (Wort/Wortform/Satz), bei der kompetente Sprecher im Blick auf (mindestens) zwei Varianten ( $\mathrm{a}, \mathrm{b}$...) in Zweifel geraten können, welche der beiden Formen (standardsprachlich) korrekt ist". Faktisch lassen sich solche Zweifel unterschiedlich nachweisen: So legt die in der Internetplattform der RatgeberCommunity gutefrage.net gestellte Frage (sowie auch die Antworten) Unsicherheiten bei der Variantenwahl offen.

(1) Frage von haecor (10.7.2007) ${ }^{1}$

Genitiv-s: Ist das „„“ davor grundsätzlich erlaubt oder nur in Ausnahmefällen?

Wikipedia schreibt dazu: „In einigen Fällen kann ein „flüchtiges e“ auftreten. Dann sind zwei Genitivvarianten möglich. Beispiel: des Baums/des Baumes." Daraus werde ich nicht so richtig schlau.

Ich meine mich zu erinnern, dass man das „e" nicht setzen soll, wenn nicht notwendig (z.B. „des Darms“ statt „des Darmes“ aber „des Mordes“, weil es sonst schwierig auszusprechen wäre.

Weiß jemand Bescheid?

(2) Antwort von critter (10.7.2007)

So kenne ich es auch. Wenn es sich gut sprechen lässt, kann man das „„“ weglassen. Aber Mords wird doch auch häufig benutzt statt Mordes. Allerdings würde ich, wenn ich einen Brief schreibe oder auch Schüler einen Aufsatz o.ä. schreiben, eher mehr zu der Form mit „e" neigen, es klingt eleganter und gebildeter. Nur in der Umgangssprache würde ich es weglassen bis auf die wenigen Ausnahmen bei schwieriger Aussprache.

\section{(3) Antwort von sdeleo (29.6.2010)}

Wenn ich mich recht erinnere, so wird ein e eingeschoben, wenn es sich beim Substantiv um ein einsilbiges Wort handelt: „des Baumes“,aber „des Tannenbaums“, weil es sich um ein mehrsilbiges Wort handelt.

In dieser Frage (und in den ausgewählten Antworten) sind - und dieser Aspekt des Zweifelns soll hier im Vordergrund stehen - laienlinguistische Erklärungen mitgeliefert, die die Wahl einer der beiden Varianten zumindest in (konzeptuell) schriftlicher Kommunikation beeinflussen (können). Sie werden in 2.1 diskutiert. Aus der linguistischen Perspektive sind die Erklärungen nicht vollständig, was u.a. an der Komplexität der Distribution beider Genitivvarianten liegt, die zwischen obligatorischer Setzung (Nullvariation), gradueller und freier Variation oszilliert (siehe dazu 2.2). Nicht

Zur Frage der Userin und den dazugehörigen Antworten siehe www.gutefrage.net/frage/genitivs-ist-das-e-davor-grundsaetzlich-erlaubt-oder-nur-in-ausnahmefaellen (Stand: 12.3.2013). 
in allen Kontexten sind beide Genitivvarianten korrekt. Es gibt also Bereiche, in denen der Sprecher nicht zweifeln muss. Dies ist das Ergebnis einer historischen Entwicklung, die im Mittelhochdeutschen angestoßen wurde und im Frühneuhochdeutschen zum formalen Umbau führte (siehe 2.3).

\subsection{Die Suche nach einfachen Regeln}

Das generelle Paradoxon, mit dem sich die Sprachbenutzer offensichtlich konfrontiert sehen, besteht aus ihrer Perspektive darin, dass von den beiden Genitivendungen, die stilistisch nicht gleichwertig sind, gerade diejenige, die „eleganter und gebildeter" klingt, eher in Ausnahmefällen verwendet werden darf, siehe die Antwort von critter in (2). Da die Genitivendungen mit unterschiedlichen Wertungen verknüpft sind (-es gilt als elegant und gebildet, $-s$ hingegen als alltäglich und umgangssprachlich), entsteht hier der Eindruck, dass der Sprachwandel zum Sprachverfall führt.

Diese wertegebundene Variation kann zur Selbstdarstellung und Abgrenzung von anderen genutzt (und so auch gefestigt) werden, zumal mit der Verwendung der langen Genitivendung hohes Stilniveau signalisiert werden kann. Der Anspruch, eine hohe Kompetenz im Bereich konzeptioneller Schriftlichkeit (meist wie in (2) auf das Schriftmedium reduziert) erworben zu haben, wirkt sich nicht nur auf die Genitivvariation aus, sondern kann sogar die Richtung des Sprachwandels bestimmen (siehe Abschnitt 3).

In der in (1)-(3) zitierten Auseinandersetzung mit der Genitivvariation auf gutefrage.net werden folgende Regeln formuliert:

1) Aussprache: Die kurze Genitivendung erschwere die Aussprache. Daher solle sie nach komplexen finalen Konsonantengruppen vermieden werden.

2) Konzeptionelle Schriftlichkeit (reduziert auf das Schriftmedium): Die kurze Genitivendung könne im Schriftverkehr (Schulaufsätze, Briefe usw.) durch die lange ersetzt werden.

3) Morphologische Komplexität (reduziert auf die Wortlänge): Die lange Genitivendung werde in mehrsilbigen Wörtern nicht verwendet. Die unterschiedliche Wortlänge in den Beispielen von sdeleo ergibt sich aus der morphologischen Komplexität der Flexionsbasis.

4) Varianz und Frequenz: Die formulierten Regeln seien nicht zuverlässig. Bei vielen Wörtern treten beide Genitivendungen auf, wobei eine der beiden vermutlich seltener sei, z.B. Mordes häufiger als Mords.

Die explizite Stigmatisierung der kurzen Genitivendung wie in Punkt 1) und 2) hat eine lange Tradition. Sie lässt sich bis ins 18. Jahrhundert zurückverfolgen. So bevorzugt Johann Christoph Adelung im Umständlichen Lehrgebäude die $e$-haltigen Genitiv- (und Dativ-)Endungen als Ausdruck der „edleren und feierlichen Schreibart" und stellt sie den synkopierten (bzw. apokopier- 
ten) Formen gegenüber, die er mit „härtern Mundarten“ und „der Sprache der Vertraulichkeit und des gemeinen Lebens" assoziiert. Allerdings bemerkt er zum Schluss selbst, dass nach der ,verbissenen“ Form durchaus aus ästhetischen Gründen „um des Wohllautes willen“ gegriffen werden kann. Die kurze Genitivendung lässt sich also nicht auf die Domäne des Umgangssprachlichen beschränken.

Das e ist in derselben [Deklinationsklasse; R.S.] ein characteristischer Biegungslaut, daher derselbe, in eigentlich Deutschen Wörtern, im Genitiv und Dativ der Einheit nie verbissen werden sollte, so häufig solches auch theils in härtern Mundarten, theils in der Sprache der Vertraulichkeit und des gemeinen Lebens der Hochdeutschen geschiehet, wo man Baums, Arms, Oheims, Wohls, Aufschubs, Abends u.s.f. für die richtigern Baumes, Armes, Oheimes, Wohles, Aufschubes, Abendes, schreibt und spricht. Wenigstens sollte die edlere und feierliche Schreibart, die dichterische, um des Silbenmaßes willen, allenfalls ausgenommen, sich dergleichen Verbeissungen nicht zu Schulden kommen lassen, es müßte denn um des Wohllautes willen geschehen. (Adelung 1971 [1782], S. 399, \178)

Noch im 19. Jahrhundert äußert sich Jacob Grimm deutlich abwertend über die kurze Genitivform:

Genitive wie prîss (?priss) f. prises, âbents f. âbendes [...] vriunts f. vriundes etc. sind nicht nachzuahmen. (Grimm 1822, S. 669)

Der stigmatisierende Charakter dieser Äußerungen wird noch einmal deutlicher, wenn sie dem tatsächlichen damaligen Sprachgebrauch gegenübergestellt werden, in dem die kurze Genitivendung (hier in schriftsprachlichen Belegen) überwog (siehe 2.3).

Aus sprachhistorischer Perspektive ist nicht allein die Tatsache interessant, dass sich die stigmatisierende Haltung (auch entgegen dem tatsächlichen Sprachgebrauch) über Jahrhunderte halten konnte, sondern auch, dass sie, ähnlich wie in anderen Fällen (siehe Davies/Langer 2006), den Verlauf des Sprachwandels mitbestimmt hat. Hierbei kommt dem Sprecher selbst, den Hundt (2009) als den Sprachsouverän bezeichnet, eine wichtige Rolle zu: Indem er Formen auswählt, wirkt er sprachnormverbreitend. Im Falle von wertegebundenen Dubletten (wie den Genitivendungen) ist dies zumindest zum Teil eine bewusste Entscheidung. Beeinflusst wird sie dadurch, dass sich der Sprecher über eigenes fehlendes oder eingeschränktes Wissen bewusst ist. Er ist, wie Antos (2003) es formuliert, das „Subjekt des Zweifels“. Wenn er sprachlich unsicher ist, sucht er nach plausiblen Erklärungen und beschreibbaren Regeln, die aus verlässlichen Quellen stammen.

Die Wirkung der Normautoritäten, die nach Davies/Langer (2006, S. 74) für die Durchsetzung von Normen oder eher Normvorstellungen von zentraler Bedeutung sind, kann auch im Falle des Genitivs angenommen werden. Dass die Korrektoren (in erster Linie Lehrer, aber auch Eltern) die Wertung (durch bevorzugte Verwendung einer) der Varianten prägen, zeigt sich indirekt darin, dass sich die User in ihren Antworten nicht explizit 
auf eine Grammatik beziehen, sondern sich vielmehr (wohl aus dem Unterricht) erinnern oder es so kennen. Auf der Suche nach einfachen Regeln bevorzugen sie möglicherweise populäre Ratgeber.

\subsection{Der Genitiv zwischen Norm und Variation}

Die formale Variation zwischen -es und $-s$ im heutigen Standarddeutschen lässt sich nicht mit einfachen Regeln beschreiben. Selbst die umfangreiche Beschreibung in der Duden-Grammatik (2009, \$\$ 301-306) ist nicht vollständig. Auf die Distribution wirken sich mehrere miteinander interagierende Faktoren aus, die unterschiedliche Variationsgrade hervorrufen:

1) Die phonologisch-prosodischen Steuerungsprinzipien betreffen den Stammauslaut und die prosodische Ausgangs- sowie Zielstruktur der Wortform. Daraus ergeben sich phonologisch definierte Wortschatzbereiche, in denen eine der beiden Varianten obligatorisch ist (Null-Variation) oder überwiegt (graduelle Variation) bzw. beide Varianten gleichermaßen möglich sind (freie Variation):

Null-Variation: Ausschließlich -es folgt auf Substantive, die auf [s] auslauten, z.B. Satz-es ['zatsos]. Kurzes $-s$ ist hingegen obligatorisch nach zwei- und mehrsilbigen, nicht finalbetonten (auch vollvokalisch auslautenden) Stämmen, z.B. A.bend-s ['a:.bənts], Ki.no-s ['ki:.nos]. Hier unterliegt die lange Genitivendung einer outputorientierten Restriktion, die dreisilbige phonologische Wörter*A.ben.d-es verhindert. Diese Restriktion betrifft daher nicht nur zweisilbige Stämme, sondern auch Suffigierungen mit Schwasilbe, z.B. Lehr+erts ['le:.res]. Darüber hinaus ist die kurze Genitivendung nach Suffigierungen mit Vollvokal obligatorisch, z.B. Reichtums (für die phonologische Analyse siehe Szczepaniak 2010).

Graduelle bis freie Variation: Zwischen den beiden Polen, in denen nur eine der beiden Endungen möglich ist, erstreckt sich der phonologischprosodisch gesteuerte Variationsbereich. Die Untersuchung geschriebener Korpora (W-Archiv des IDS Mannheim) in Szczepaniak (2010) ergab folgende Variation: Einsilbige Simplizia weisen die stärkste Tendenz zur langen Genitivendung auf, morphologisch und phonologisch komplexe Substantive tendieren hingegen zur kurzen Endung: Die relative Frequenz der kurzen Endung liegt bei Simplizia bei 0,37, während sie bei Derivaten mit unbetontem Präfix, z.B. Verstand, 0,67 und bei Derivaten mit betontem Präfix, z.B. Anruf, sogar 0,78 beträgt. Komposita weisen tendenziell einen höheren Anteil an kurzen Genitivendungen auf als Simplizia, z.B. Stands $(0,06)$ vs. Komposita mit Stand als Zweitglied, z.B. Kontostand $(0,28)$ (siehe aber Punkt 5 zur semantischen Transparenz von Komposita). 
Zum anderen ist die Variantenwahl von der Qualität und Quantität des Wortauslauts abhängig: Je sonorer der Wortauslaut, desto häufiger die kurze Genitivendung, z.B. des Lärms, aber des Bett(e)s. Sie tritt umso seltener auf, je komplexer die wortauslautende Konsonantengruppe, z.B. eher des Betts als des Pferds. Bei vokalisch auslautenden Einsilblern sowie bei mehrsilbigen Wörtern mit finalbetontem Vollvokal nimmt die Duden-Grammatik (2009, \$S 301-306) eine starke Tendenz zur kurzen Genitivendung an.

2) Lexikalische Steuerungsprinzipien (graduelle bis freie Variation): Die lange Genitivendung tritt v.a. bei Erb- und Lehnwörtern auf, nur selten bei Fremdwörtern (und Eigennamen) - hier aber immer beim s-Auslaut, z.B. des Atlasses, neben des Atlas (siehe Duden-Grammatik 2009, SS 301-306).

3) Frequenz (graduelle bis freie Variation): Bei sehr frequenten (einsilbigen) Substantiven, z.B. Gott, wird eine der beiden Endungen (hier: Gottes) deutlich präferiert, während weniger frequente, z.B. Spott, beide Varianten (Spottes und Spotts) zulassen (Fehringer 2004).

4) Morphologische Kriterien (Null-Variation): Substantivierungen, z.B. des Rots, des Wenns, verlangen die kurze Endung (oder sind sogar endungslos). Auch bei Kurzwörtern ist die lange Endung ausgeschlossen, z.B. des LKWs (Duden-Grammatik 2009, SS 314-315).

5) Morphologische Transparenz (graduelle Variation): Semantisch transparente Komposita, z.B. Blindenbundes, tendieren nach Fehringer (2011) zu derselben Genitivvariante wie die entsprechenden Simplizia (Hundes). Opake Komposita wie Seehund weichen davon ab.

\subsection{Genitivendungen im Frühneuhochdeutschen}

Historisch gesehen ist die kurze Genitivendung die jüngere. Sie entstand erst durch die mittelhochdeutsche Synkope, die zunächst nur in bestimmten prosodischen Kontexten möglich war:

1) Bereits im Mittelhochdeutschen entwickelte sich die Tendenz zur $e$-Tilgung in dreisilbigen Flexionsformen, z.B. mhd. fa.te.res $>$ fa.ters oder en.ge.les $>$ en.gels. Dieser Prozess trug zum typologischen Wandel des Deutschen von einer Silben- zu einer Wortsprache bei, weil sich damit die im Gegenwartsdeutschen starke Präferenz des trochäischen Wortes herausgebildet hat (siehe Szczepaniak 2007, 2012). Heute gibt es bei zweisilbigen Stämmen keine Variation; hier ist $-s$ obligatorisch, z.B. des Segels, des Vaters, des Bodens, des Abends. 
2) Die Tilgung fand im Mittelhochdeutschen auch nach einsilbigen Stämmen statt, doch zunächst nur nach Liquiden (nach Kurzvokal), z.B. spiles > spils ,Spiel-GEN.SG..

Anschließend weitete sich die Synkope aus, so dass zum einen der Sonoritätsunterschied zwischen finalem Konsonanten und Genitiv-s immer geringer wurde, bis schließlich im Frühneuhochdeutschen das extrasilbische (Genitiv)-s aufkam, z.B. in nhd. Flugs [flu:ks]. Zum anderen dehnte sich $-s$ auch auf Stämme mit langem Vokal und solche mit auslautenden Konsonantenclustern aus (siehe unten).

Die fortschreitende Überwindung der anfänglichen phonologisch-prosodischen Beschränkungen führte zum Frequenzanstieg der kurzen Genitivendung im Frühneuhochdeutschen. Die Analyse des Bonner Frühneuhochdeutschkorpus (www.korpora.org/Fnhd/) ergibt ein deutliches Bild des sich vollziehenden Normwandels (siehe Tabelle 1). ${ }^{2}$ Bei den einsilbigen Simplizia lässt sich eine graduelle Frequenzverschiebung von -es (graue Balken in Abb. 1), das im Zeitraum 1350-1400 mit 71\% noch überwiegt, zum -s (schwarze Balken in Abb. 1), das zwischen 1650 und 1700 denselben Wert erreicht, feststellen.

\begin{tabular}{|l|r|r|r|r|}
\hline & $1350-1400$ & $1450-1500$ & $1550-1600$ & $1650-1700$ \\
\hline$-e s$ & $71 \%(292)$ & $43 \%(160)$ & $43 \%(205)$ & $26 \%(102)$ \\
\hline$-s$ & $26 \%(108)$ & $56 \%(210)$ & $56 \%(266)$ & $74 \%(292)$ \\
\hline$\varnothing$ & $2 \%(10)$ & $1 \%(4)$ & $1 \%(4)$ & $<1 \%(1)$ \\
\hline Gesamt & 410 & 374 & 475 & 395 \\
\hline
\end{tabular}

Tab. 1: Frequenz beider Genitivendungen im Frühneuhochdeutschen (Datenbasis: Das Bonner Frühneuhochdeutschkorpus)

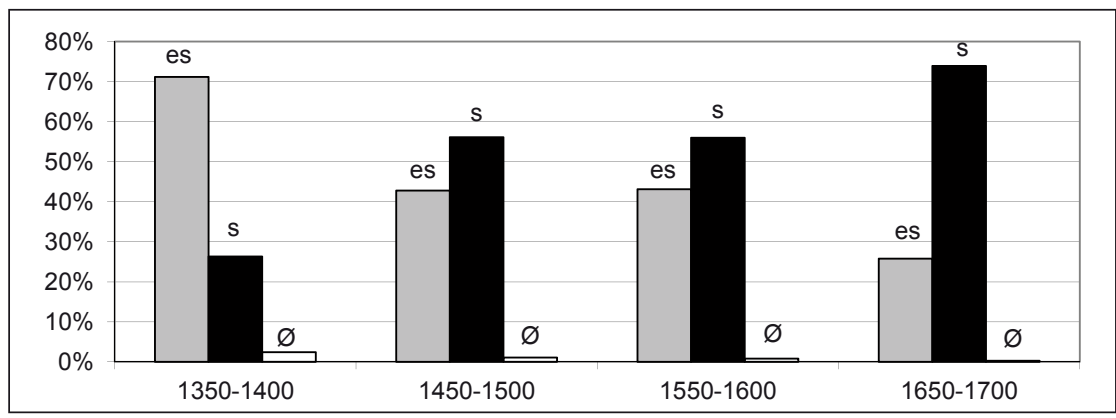

Abb. 1: Frequenz beider Genitivendungen im Frühneuhochdeutschen (Datenbasis: Das Bonner Frühneuhochdeutschkorpus)

2 Die folgende Analyse umfasst Teste aus dem obersächsischen, ostfränkischen, hessischen und ripuarischen Gebiet. 
Die Ergebnisse der Korpusuntersuchung zeigen, dass die Durchsetzung der kurzen Genitivendung silbenphonologisch gesteuert war: Im ersten Zeitraum 1350-1400 (Abb. 2) tritt -s nach ein- und zweigliedrigen Konsonantengruppen auf, zwischen 1650-1700 (Abb. 3) auch nach dreigliedrigen. Diese Tendenz stimmt mit dem generellen Zuwachs an komplexen Konsonantenclustern überein, den Werner (1978) in der Geschichte des Deutschen nachweist.

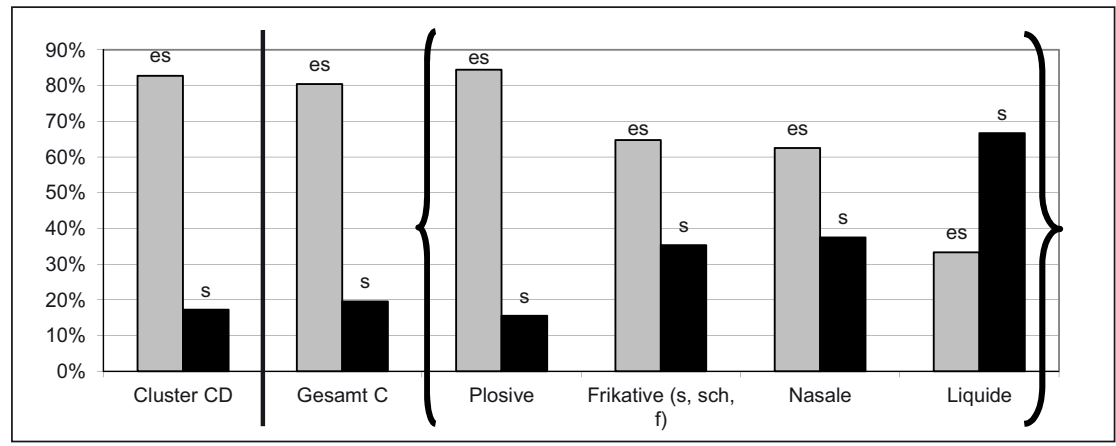

Abb. 2: Distribution beider Genitivendungen im Zeitraum 1350-1400

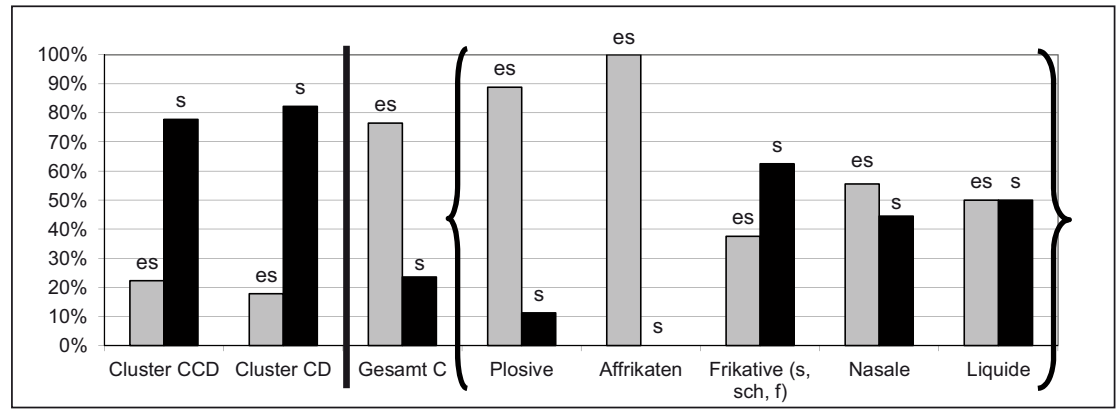

Abb. 3: Distribution beider Genitivendungen im Zeitraum 1650-1700

Nach einfach geschlossenen Silben steigt zwischen 1350 und 1700 der Anteil an kurzen Genitivendungen sonoritätsgesteuert an. Dass Plosive eher als (sonorere) Affrikaten den Übergang zur kurzen Endung fördern, kann mit der phonetischen Komplexität der Affrikaten, z.B. [pf], erklärt werden, denn hierbei entstehen dreigliedrige Auslautcluster, z.B. Kopfs. Bei [ts] trägt das morphologische Konstanzprinzip zur Erhaltung von -es bei, z.B. Satzes.

3) Aufgrund der zu geringen Datenbasis können keine zuverlässigen Aussagen über die Rolle der morphologischen und phonologischen Komplexität bei der Durchsetzung von $-s$ gemacht werden. Es ist jedoch erwähnenswert, dass im Zeitraum 1350-1400 unter den 23 Komposita mit einsilbigem Kopf lediglich in fünf Fällen die kurze Genitivendung erscheint. So 
schwankt beispielsweise die Genitivendung von $<$ hymelr(e)ich $>$ zwischen $<$ hymelr(e)iches> (3 Mal) und <hymelr(e)ichs> (5 Mal). Im letzten Untersuchungszeitraum 1650-1700 überwiegt schon die kurze Genitivendung.

Diese kleine diachrone Studie zeigt, dass sich viele der heutigen Variablen bereits im Frühneuhochdeutschen abzeichnen und den Normwandel zur kurzen Genitivendung fördern. Es steht zu vermuten, dass dieser durch die soziolinguistische Ausdifferenzierung (Stigmatisierung) der Varianten, d.h. im Zuge der Entwicklung der überregionalen normierten Schriftsprache, aufgehalten wurde/wird. Die den Sprachwandel aufhaltende Polarisierung dürfte mit der Einführung der Schulpflicht seit dem 19. Jahrhundert zugenommen haben. Vermutlich trugen die Korrektoren als Norminstanz zur Verbreitung von soziolinguistisch aufgeladenen Normen (der stilistische Mehrwert des langen Genitivs) bei, sicherlich dienten auch die Bildungsträger in ihrer sozialen Bestätigungs- und Aufwärtsbestrebung als Multiplikatoren.

\section{Funktionaler Umbau des Genitivs}

Eine weit größere Auswirkung der soziolinguistischen Kontrastierung von Varianten kann im Bereich des funktionalen Wandels beobachtet werden. Hier ist neben dem Abbau des verbalen und attributiven Genitivs ein unerwarteter Aufbau des Genitivs als Präpositionalkasus von sekundären Präpositionen wie dank, entgegen, entsprechend oder gemäß zu beobachten. Diese ursprünglichen Dativpräpositionen wechseln zum Genitiv, z.B. dank dem guten Wetter und dank des guten Wetters (für eine umfangreiche Korpussammlung siehe Di Meola 2000). Bei der Präposition trotz ist der Rektionswandel bereits fast abgeschlossen.

\subsection{Genitiv als Marker für fortschreitende Grammatikalisierung sekundärer Präpositionen}

In der Grammatikalisierungsforschung gehört die Kasuswahl zum wichtigen Kriterium, nach dem der Grammatikalisierungsgrad von Präpositionen bestimmt wird, d.h. Grammatikalisierung äußert sich im Rektionswandel. Im Deutschen regieren prototypische, d.h. kurze, nicht-segmentierbare, semantisch und syntaktisch vielwertige Präpositionen den Dativ bzw. den Akkusativ (siehe Lindqvist 1994). Zu Gunsten dieser Kasus geben sekundäre (jüngere und morphologisch transparente) Elemente im Zuge ihrer fortschreitenden Grammatikalisierung die Genitivrektion auf. Hier ist also Variation zu erwarten, wohingegen tertiäre Präpositionen wie in Bezug auf ihre Rektion via primäre Präposition (hier: auf) abwickeln: 
Rektion via primäre Präposition
Genitiv/Dativ/

Genitiv Akkusativ-Variation

entlang, während, wegen $\quad$ auf, bei, zu
Akkusativ bzw. Dativ

zunehmender Grammatikalisierungsgrad

Die kausale Präposition wegen (ähnlich auch temporales wäbrend und lokales entlang) ist in ihrer Entwicklung dicht an die primären Präpositionen gerückt. Die kausale Bedeutung, in der sie seit dem 14./15. Jahrhundert verwendet wurde, setzte sich im 19. Jahrhundert durch (siehe Braunmüller 1982; Lindqvist 1994; Szczepaniak 2011). Ihre formale Entwicklung von X wegen $>X$ wegen $>$ wegen $X$ umfasste neben der Ausdrucksreduktion auch den Stellungswandel von der Zirkum- über die Post- zur Präposition. Schließlich war seit dem 17. Jahrhundert neben der (ursprünglichen) Genitiv- auch die Dativselektion möglich (Davies/Langer 2006, S. 200) (zum Umgang mit der variablen Kasusrektion in den historischen Grammatiken siehe Abschnitt 3.2).

Eine gegenläufige Entwicklung weisen sekundäre Präpositionen auf, darunter entgegen, entsprechend, nahe und gemäß, die ihre ursprüngliche Dativrektion zugunsten des Genitivs aufgeben. Di Meola (1999, 2000, 2004) erklärt diesen Trend damit, dass bei der Grammatikalisierung ein maximaler Abstand zum Spenderlexem gesucht wird. Da diese dem Bezugssubstantiv folgen, z.B. Sie ist dem Ziel (sehr) nahe, wird durch den Stellungswandel und den Rektionswechsel vom Dativ zum Genitiv hin der funktionale Unterschied zum Spenderlexem formal verdeutlicht, z.B. Sie ist nahe dem Ziel > Sie ist nahe des Ziels. Die Tendenz zur Genitivrektion ist dabei umso stärker, je fortgeschrittener der Stellungswandel von der Nach- zur Voranstellung ist, da nur Präpositionen den Rektionswechsel zulassen:

\begin{tabular}{|l|c|c|}
\hline Präposition & Anteil Voranstellung & Anteil Genitiv \\
\hline entgegen & $45 \%$ & $5 \%$ \\
\hline entsprechend & $75 \%$ & $8 \%$ \\
\hline nahe & $79 \%$ & $11 \%$ \\
\hline gemäß & $97 \%$ & $25 \%$ \\
\hline
\end{tabular}

Tab. 2: Die Korrelation zwischen Stellungs- und Rektionswandel von sekundären Präpositionen (aus: Di Meola 1999, S. 345-346)

Dieses Prinzip gelte laut Di Meola (2004) auch für sekundäre Präpositionen mit ursprünglicher Genitivrektion wie entlang oder gegenüber, die im Zuge der Grammatikalisierung zur Dativflexion tendieren. Jedoch ist diese Tendenz deutlich weniger ausgeprägt. Die Daten sprechen eher für einen gerichteten Wandel Dativ > Genitiv. 
Die auf dem Language Forum von Wordreference.com geführte Online-Diskussion (hier nur in Ausschnitten präsentiert) ${ }^{3}$ zeigt eine weitere Dimension des Rektionswandels, die es zu berücksichtigen gilt.

(4) Initiierender Beitrag von Nanexa (28.7.2007, 15:55)

Ich verwende das Wort „dank“ eigentlich immer mit Genitiv. also:

Dank des Einsatzes der Feuerwebr ...

Dank deiner Bemübungen ...

Nun hab ich heute wieder in einem Buch gelesen: dank dem schnellen Eingreifen ... und das klingt für mich so falsch.

Ich finde dank+dativ öfters - was ist denn nun richtig?

Genitiv oder Dativ?

(5) Antwort von ablativ $(28.7 .2007,16: 12)$

Lt. „Wikipedia“ und „canoo“, wo ja genau Deine Beispiele gezeigt werden, ist sowohl Gen. wie Dat. richtig. Vorzugsweise sogar eher Dativ; der Genitiv sei aber auch richtig, besonders im Plural.

(6) Antwort von Suilan (28.7.2007, 16:24)

Duden \#9, 2001, sagt allerdings, dass der Dativ im Plural eines stark gebeugten Substantivs bevorzugt wird, da der Genitiv hier den Kasus nicht deutlich machen würde. Das Beispiel: „dank Fortschritten in der Wissenschaft“.

(7) Resümee von Nanexa (28.7.2007, 16:45)

Danke für Eure Antworten.

Ich nehme dann mal an, dass es sich mit dank genauso wie mit wegen verhält. Hier war ursprünglich nur der Genitiv richtig und inzwischen ist auch der Dativ erlaubt ...

@.Suilan:

wieso, man könnte doch genauso gut sagen : dank der Fortschritte in der Wissenschaft. ?? Ohne Artikel würde ich das nicht verwenden, klingt komisch, finde ich.

(8) Antwort von Suilan (28.7.2007, 17:28)

Laut Duden war's wohl andersherum: dank kam ursprünglich von „Dank sei seinem Einfluss, “ daher Dativ, aber weil andere „unechte“ Präpositionen (d.h. solche, die aus einem Substantiv entstanden) mit Genitiv gebraucht werden, z.B. kraft, laut, statt, infolge, wurde bei dank der Genitiv auch üblich.

Ist ja auch nur gerecht so! Wenn der Dativ sonst überall den Genitiv vertreibt! ${ }^{4}$

http://forum.wordreference.com/showthread.php?t=594432 (Stand: 3.7.2013).

4 Schneider (2005, S. 158) verweist auf die wertende Formulierung von Bastian Sick, dass dem Genitiv bei der Präposition trot», „eine feindliche Übernahme gelungen“ sei. 
In dieser Diskussion wird zum einen eine Analogie zu wegen, bekannt für seine Kasusschwankung, gesucht. Zum anderen wird die Schwankung mit dem Abbau (ja der Verdrängung) des Genitivs gleichgesetzt, was den (auch zum Teil bewussten) hyperkorrekten Gebrauch des Genitivs auslöst.

\subsection{Die Stigmatisierung des Präpositionaldativs bei wegen}

Die Rektionsschwankung bei wegen ist im laienlinguistischen Bewusstsein fest etabliert. Dabei ist der Dativ auch heute noch negativ konnotiert. In der historischen Grammatikographie lässt sich eine zunehmende Stigmatisierung beobachten, die deutlich später auftritt als die Variation selbst. So stellen Davies/Langer (2006, S. 200-211) vier Stigmatisierungsphasen fest:

1) In den Grammatiken des 17. Jahrhunderts wird die bereits bestehende Variation nicht thematisiert. Wegen wird entweder als Genitiv- oder als Dativpräposition aufgeführt. Wie Davies/Langer (2006, S. 201) beobachten, weichen in Stielers „Der Teutschen Sprache Stammbaum und Fortwachs oder Teutscher Sprachschatz [...]" von 1691 sogar die Beispiele von der angegebenen Rektionsregel ab.

2) Seit 1700 wird die Präposition als genitivregierend beschrieben. Die Dativrektion bleibt unerwähnt; der Dativ wird indirekt stigmatisiert.

3) Ab der 2. Hälfte des 18. Jahrhunderts, zuerst in Heynatz' „Deutsche Sprachlehre zum Gebrauch der Schulen" von 1777, wird der Dativ explizit stigmatisiert.

4) Seit den 1980er Jahren wird die Verwendung des Dativs in Ausnahmefällen erlaubt.

Nach der Duden-Grammatik (2009, \917) gehört wegen zu vier Präpositionen, die den Dativ „erlauben, gerade in der gesprochenen Sprache“.

Die Studie von Elspaß (2005) zeigt, dass die (ältere) Genitivrektion bei wegen, aber auch während, (an) statt und weiteren Präpositionen im 19. Jahrhundert bereits zum Zeichen höherer Bildung aufgestiegen ist. Sie tritt in den Auswandererbriefen deutlich seltener auf als die Dativ-/Akkusativrektion (nur zu 26\%). Den insgesamt seltenen Genitiv verwenden über vier Fünftel der Schreibenden mit höherer Bildung, aber nur ein Fünftel mit Volksschulbildung. Daraus kann geschlossen werden, dass die (vermutlich eher implizite) Stigmatisierung des Dativs im 19. Jahrhundert an höheren Schulen weiter vorangeschritten war als an Volksschulen, was den Wert des Genitivs als Indikator einer höheren sozialen Klasse (der Bildungsschicht) verstärkte. In der Übersichtsstudie von Davies (2005) geben 57\% aller Lehrer heute noch an, dass sie die Verwendung des Dativs als umgangssprachlich einstufen. Die Dativrektion, obwohl weitestgehend akzeptiert, wird immer noch mit konzeptioneller Mündlichkeit assoziiert (siehe u.a. Schneider 2005; Zifonun/Hoffmann/Strecker 1997). 


\subsection{Genitiv bei sekundären Präpositionen}

Aufgrund von Kasusschwankungen (Genitiv/Dativ oder Akkusativ) werden sekundäre Präpositionen wie entsprechend oder dank laienlinguistisch parallel zur Präposition wegen behandelt: Wie die zitierte Online-Diskussion zeigt, leiten Sprecher von dem bekannten Fall der Präposition wegen die (sprachhistorisch inkorrekte) Regel ab, dass die kasusschwankenden Präpositionen die Genitivrektion zugunsten der Dativrektion aufgeben. Dieses sich dabei zeigende Interesse an sprachhistorischen Zusammenhängen sollte im schulischen Unterricht bedient werden. Doch wie im Folgenden in Anlehnung an Becker (2011) diskutiert wird, werden sekundäre Präpositionen nur ganz selten im Schulunterricht behandelt. Sie bleiben eine Quelle sprachlicher Unsicherheiten, da ihr Erwerb aufgrund folgender Aspekte erschwert wird (siehe Becker 2011):

1) Frequenz: Sekundäre Präpositionen haben eine viel geringere Frequenz als primäre.

2) Konzeptionelle Zuordnung: Im Gegensatz zu primären Präpositionen, die gleichermaßen in nähe- wie distanzsprachlichen Texten auftreten, gehören sekundäre Präpositionen eher der konzeptionell schriftlichen Kommunikation an. (Tertiäre Präpositionen sind noch distanzsprachlicher.)

3) Erwerbsabschluss: Der Erwerb von Präpositionen verläuft gestaffelt, was u.a. aus den Unterschieden in konzeptioneller „Gebundenheit“ resultiert. Am frühesten werden primäre Präpositionen erworben: in, auf, unter im 3. Lebensjahr, neben, hinter, vor, zwischen im 5. Lebensjahr. Der Erwerb semantisch-lexikalischer Aspekte von primären Präpositionen ist im Grundschulalter abgeschlossen, während der morphologisch-syntaktische Aspekt, u.a. die Kasusrektion, mehr Schwierigkeiten bereitet; hier mangelt es aber an entsprechenden Studien zum Erwerbsabschluss. Für den Erwerb der semantischen und syntaktischen Aspekte der sekundären und tertiären Präpositionen geht Becker (2011, S. 206) von einem langen Aneignungsprozess aus, der (insbesondere bei tertiären Präpositionen) mit dem Erwerb der konzeptionellen Schriftlichkeit zusammenhängt.

4) Schulunterricht: Gleichzeitig, dies bemängelt Becker (2011, S. 201), wird der Erwerb der drei Präpositionengruppen sprachdidaktisch unterschiedlich intensiv begleitet. In den Lehrbüchern werden die primären Präpositionen thematisiert, die sekundären nur ausnahmsweise und die tertiären überhaupt nicht.

5) Grammatikographie: Die Grammatiken liefern heterogene, zum Teil widersprüchliche Informationen zur Rektion sekundärer Präpositionen.

Wie die Studie von Becker (2011) zeigt, greifen erwachsene Sprecher im Zweifel zum Genitiv. Becker legte den Studierenden Lückentexte in zwei 
Versionen vor: in einer eher fachsprachlichen, formelleren und einer alltagssprachlichen, informelleren Version. Fast völlig unabhängig vom Formalitätsgrad entschieden sich die Probanden (wohl aufgrund der Testsituation in der Bildungsstätte) meist für den Genitiv. Neben reinen Dativpräpositionen untersucht Becker auch reine Genitiv- und kasusschwankende Präpositionen, siehe Tabelle 3.

\begin{tabular}{|l|c|c|}
\hline \multicolumn{1}{|c|}{ Untersuchte Präpositionen } & Mit Genitiv & Mit Dativ \\
\hline $\begin{array}{l}\text { Dativpräpositionen } \\
\text { (gemäß, außer, gegenüber, entsprechend, entgegen) }\end{array}$ & $65 \%$ & $35 \%$ \\
\hline $\begin{array}{l}\text { Dativ-/Genitivpräpositionen } \\
\text { (einschließlich, während, laut, innerhalb, dank) }\end{array}$ & $86 \%$ & $14 \%$ \\
\hline $\begin{array}{l}\text { Genitivpräpositionen } \\
\text { (bezüglich, ungeachtet, hinsichtlich, längs, jenseits) }\end{array}$ & $97 \%$ & $3 \%$ \\
\hline
\end{tabular}

Tab. 3: Der Anteil an Genitiv- und Dativrektion in der Studie von Becker (2011)

Die Studie zeigt, dass der Genitiv bei sekundären Präpositionen vorherrscht. Damit liefert Becker (2011) ein alternatives Szenario für die Entwicklung der sekundären Präpositionen. Während Di Meola (siehe Abschnitt 3.1) einen auf maximale Distanz zur lexikalischen Quelle gerichteten Grammatikalisierungspfad annimmt, der gleichermaßen den Rektionswandel Genitiv $>$ Dativ und Dativ > Genitiv umfasst, sprechen die Ergebnisse aus Becker (2011) dafür, dass bei den distanzsprachlich assoziierten sekundären Präpositionen der prestigehaltigere Kasus gewählt wird. Hierdurch können sich Sprecher als der Bildungsschicht zugehörig darstellen. Diese soziolinguistisch beeinflusste Wahl lenkt den Sprachwandel und fördert die Verwendung des Genitivs (vgl. Labov 1978, 2001).

\section{Fazit}

Sprachliche Unsicherheiten beeinflussen den Sprachwandel. Sie können ihn anhalten oder sogar lenken. Der Normwandel von der langen zur kurzen Genitivendung, der durch die phonologisch-typologische Neuorientierung des Deutschen in Gang gesetzt wurde, ist vermutlich durch die stilistische Aufwertung der langen Genitivendung aufgehalten worden.

Die mediale und sprachkonzeptuelle Aufwertung des Genitivs als Präpositionalkasus lässt sich bereits für das frühe Neuhochdeutsche belegen. Auf diese kann der Rektionswandel Dativ $>$ Genitiv bei sekundären Präpositionen zurückgeführt werden, zumal bei sprachlicher Unsicherheit der Prestigegenitiv bevorzugt wird. 


\section{Literatur}

Adelung, Johann Christoph (1971 [1782]): Umständliches Lehrgebäude der deutschen Sprache zur Erläuterung der deutschen Sprachlehre für Schulen. Bd. 1. (= Documenta linguistica: Reihe 5, Deutsche Grammatiken des 16. Und 18. Jahrhunderts). Hildesheim/New York. [Reprogr. Nachdruck d. Ausg. Leipzig 1782.]

Antos, Gerd (2003): „Imperfektibles“ sprachliches Wissen. Theoretische Vorüberlegungen zu „sprachlichen Zweifelsfällen“. In: Linguistik online 16, S. 35-46.

Becker, Tabea (2011): „Entgegen des Trends“: Erwerb, Rektion und Didaktik von Präpositionen. In: Köpcke, Klaus-Michael/Noack, Christina (Hg.): Sprachliche Strukturen thematisieren. Sprachunterricht in Zeiten der Bildungsstandards. (= Diskussionsforum Deutsch 28). Baltmannsweiler, S. 199-217.

Braunmüller, Kurt (1982): Syntaxtypologische Studien zum Germanischen. (= Tübinger Beiträge zur Linguistik 197). Tübingen.

Davies, Winifred (2005): Deutschlehrer und Deutschlehrerinnen (in Deutschland) als Geber und Vermittler von sprachlichen Normen. In: Deutscher Akademischer Austauschdienst (DAAD) (Hg.): Germanistentreffen: Deutschland - Großbritannien - Irland. Dresden 30.9.-3.10.2004. Dokumentation der Tagungsbeiträge. Bonn, S. 323-338.

Davies, Winifred V./Langer, Nils (2006): The making of bad language. Lay linguistic stigmatisations in German: past and present. (= VarioLingua 28). Frankfurt a.M. u.a.

Di Meola, Claudio (1999): Entgegen, nahe, entsprechend und gemäß. Dativpräpositionen mit Genitivrektion. In: Zeitschrift für Germanistische Linguistik 27, S. 344-351.

Di Meola, Claudio (2000): Die Grammatikalisierung deutscher Präpositionen. (= Studien zur deutschen Grammatik 62). Tübingen.

Di Meola, Claudio (2004): The rise of the prepositional genitive in German - a grammaticalization phenomenon. In: Lingua 114, S. 165-182.

Duden-Grammatik (2009): Der Duden. Bd. 4: Die Grammatik. Unentbehrlich für richtiges Deutsch. 8., überarb. Aufl. Hrsg. v. d. Dudenredaktion. Mannheim/Wien/ Zürich.

Elspaß, Stephan (2005): Standardisierung des Deutschen. Ansichten aus der neueren Sprachgeschichte, von unten. In: Eichinger, Ludwig M./Kallmeyer, Werner (Hg.): Standardvariation. Wie viel Variation verträgt die deutsche Sprache? (= Jahrbuch des Instituts für Deutsche Sprache 2004). Berlin/New York, S. 63-99.

Fehringer, Carol (2004): How stable are Morphological Doublets? A case study of $/ \partial / \sim \varnothing$ variants in Dutch and German. In: Journal of Germanic Linguistics 16, S. $285-329$.

Fehringer, Carol (2011): Allomorphy in the German genitive. A paradigmatic account. In: Zeitschrift für germanistische Linguistik 39, S. 90-112.

Grimm, Jacob (1822): Deutsche Grammatik. Erster Theil. 2. Ausg. Göttingen.

Hundt, Markus (2000): „Spracharbeit“ im 17. Jahrhundert. Studien zu Georg Philipp Harsdörffer, Justus Georg Schottelius und Christian Gueintz. Berlin/New York. 
Hundt, Markus (2009): Normverletzungen und neue Normen. In: Konopka, Marek/ Strecker, Bruno (Hg.): Deutsche Grammatik - Regeln, Normen, Sprachgebrauch. (= Jahrbuch des Instituts für Deutsche Sprache 2008). Berlin/New York, S. 117140 .

Klein, Wolf Peter (2003): Sprachliche Zweifelsfälle als linguistischer Gegenstand. Zur Einführung in ein vergessenes Thema der Sprachwissenschaft. In: Linguistik online 16, 4, S. 5-33. Internet: www.linguistik-online.de/16_03/klein.pdf (Stand 30.10.2013).

Labov, William (1978): Sprache im sozialen Kontext: Beschreibung und Erklärung struktureller und sozialer Bedeutung von Sprachvariation. Bd. 2. Hrsg. von Norbert Dittmar und Bert-Olaf Rieck. (= Monographien Linguistik und Kommunikationswissenschaft 23). Königstein i.Ts.

Labov, William (2001): Principles of linguistic change. Bd. 2: Social factors. (= Language in Society 29). Malden, MA u.a.

Lindqvist, Christer (1994): Zur Entstehung von Präpositionen im Deutschen und Schwedischen. (= Linguistische Arbeiten 311). Tübingen.

Schneider, Jan Georg (2005): Was ist ein sprachlicher Fehler? Anmerkungen zu populärer Sprachkritik am Beispiel der Kolumnensammlung von Bastian Sick. In: Aptum. Zeitschrift für Sprachkritik und Sprachkultur 2005, S. 154-177.

Szczepaniak, Renata (2007): Der phonologisch-typologische Wandel des Deutschen von einer Silben- zu einer Wortsprache. (= Studia Linguistica Germanica 85). Berlin/ New York.

Szczepaniak, Renata (2010): Während des Flug(e)s/des Ausflug(e)s: German short and long genitive endings between norm and variation. In: Lenz, Alexandra/Plewnia, Albrecht (Hg.): Grammar between norm and variation. (= VarioLingua 40). Frankfurt a.M., S. 103-126.

Szczepaniak, Renata (2011): Grammatikalisierung im Deutschen. Eine Einführung. 2., überarb. u. erw. Aufl. Tübingen.

Szczepaniak, Renata (2012): Lautwandel verstehen. Vom Nutzen der Typologie von Silben- und Wortsprachen für die historische und die synchrone germanistische Linguistik. In: Maitz, Péter (Hg.): Historische Sprachwissenschaft: Erkenntnisinteressen, Grundlagenprobleme, Desiderate. (= Studia Linguistica Germanica 110). Berlin/New York, S. 85-104.

Werner, Otmar (1978): Schwa-Schwund und Phonotaktik im Deutschen. In: Birnbaum, Henrik et al. (Hg.): Studia linguistica Alexandro Vasilii filio Issatschenko a collegis amicisque oblata. Lisse, S. 127-147.

Zifonun, Gisela/Hoffmann, Ludger/Strecker, Bruno (1997): Grammatik der deutschen Sprache. 3 Bde. (= Schriften des Instituts für Deutsche Sprache 7). Berlin/New York. 
